Supporting Information

\title{
Evaluation of Chromophore and Assembly Design in Light-Driven Water Splitting with a Molecular Water Oxidation Catalyst
}

\author{
Matthew V. Sheridan ${ }^{\dagger}$, Benjamin D. Sherman ${ }^{\dagger}$, Rodolfo L. Coppo ${ }^{\ddagger}$, Degao Wang ${ }^{\dagger}$, Seth L. \\ Marquard $^{\dagger}$, Kyung-Ryang Wee ${ }^{\dagger}$, Neyde Y. Murakami Iha ${ }^{\ddagger}$, and Thomas J. Meyer ${ }^{\dagger *}$ \\ ${ }^{\dagger}$ Department of Chemistry, University of North Carolina at Chapel Hill, Chapel Hill, NC 27599
} (USA)

\$Laboratory of Photochemistry and Energy Conversion, Departamento de Química Fundamental, Instituto de Química, Universidade de São Paulo - USP, Av. Prof. Lineu Prestes, 748, 05508-000 São Paulo, SP, Brazil.

Email: tjmeyer@unc.edu

\section{Experimental}

The solar to hydrogen (STH) conversion efficiency was found using equation S1 which takes into account Faradaic efficiency of $\mathrm{H}_{2}$ production $\left(\eta_{\mathrm{H} 2}\right)$, overpotential or applied bias $\left(\mathrm{V}_{\text {app }}\right)$, current $(A)$, and light intensity (I). For this experiment, the cell was illuminated using white light illumination at an intensity of $100 \mathrm{~mW} \mathrm{~cm}^{-2}$, at an overpotential of $600 \mathrm{mV}$ against a Pt electrode under $\mathrm{N}_{2}$ (Pt was used as the counter and reference electrode), and the $\mathrm{H}_{2}$ production was assumed to be $100 \%$ at the Pt counter electrode. This setup mirrors experiments previously validated in our laboratory. ${ }^{1}$ At the best operating electrode (RuP$\mathbf{H}^{2+}-1, \sim 1.5 \mathrm{~mA} / \mathrm{cm}^{2}$ ) the STH obtained was $\sim 1 \%$.

$$
\operatorname{STH}(\%)=\frac{A V_{a p p} \eta_{H_{2}}}{I}
$$

Catalyst $^{2}$ and chromophores ${ }^{3,4,5}$ were synthesized as previously described. Fluorine-doped tin oxide (FTO) was used as the back contact of the photoanodes and was purchased from Hartford Glass $(15 \Omega / \mathrm{sq})$. SnO2 films were prepared as previously described and their thickness measured with a profilometer. ${ }^{6}$ Atomic layer deposition (ALD) was performed using a Cambridge NanoTech Savannah S200 instrument with TDMAT (tetrakis(dimethylamino)titanium) as Ti precursor with shell thicknesses determined by TEM on Si wafers. Electrochemical and photoelectrochemical experiments were performed as previously described. ${ }^{1}$ 


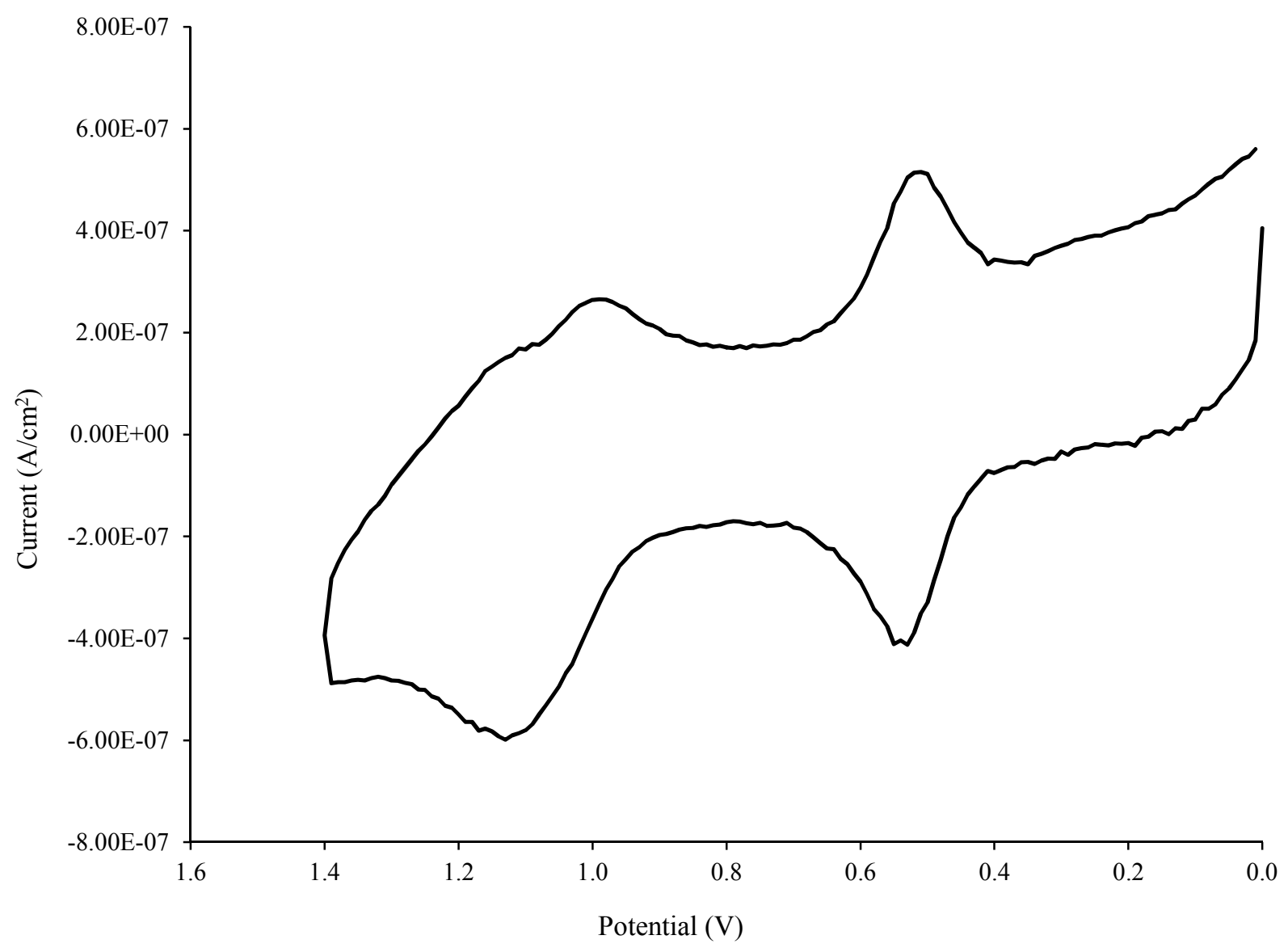

Figure S1. CV of RuP-H ${ }^{2+}$ co-loaded with $\mathbf{1}$ at a ratio of 2:1 on planar FTO in a pH 1, 0.1 $\mathrm{M} \mathrm{HClO}_{4}, \mathrm{~V}$ vs. $\mathrm{Ag} / \mathrm{AgCl}, \mathrm{v}=0.025 \mathrm{~V} / \mathrm{s}$. 


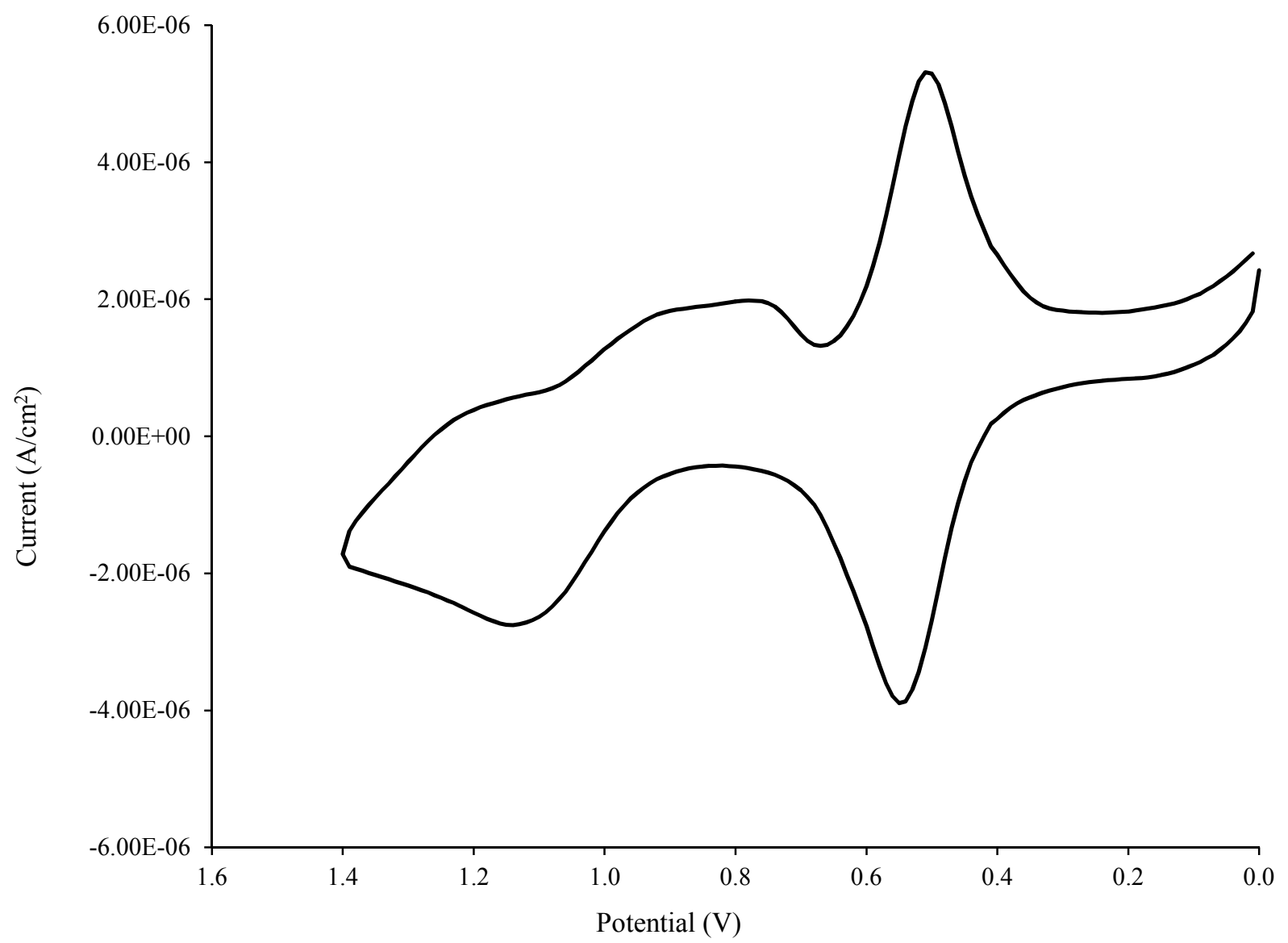

Figure S2. CV of $-\mathbf{R u P}_{2}{ }^{2+}-\mathrm{Zr}(\mathrm{IV})-\mathbf{1}$ on planar FTO on planar FTO in a pH 1, 0.1M $\mathrm{HClO}_{4}, \mathrm{~V}$ vs. $\mathrm{Ag} / \mathrm{AgCl}, \mathrm{v}=0.025 \mathrm{~V} / \mathrm{s}$. 


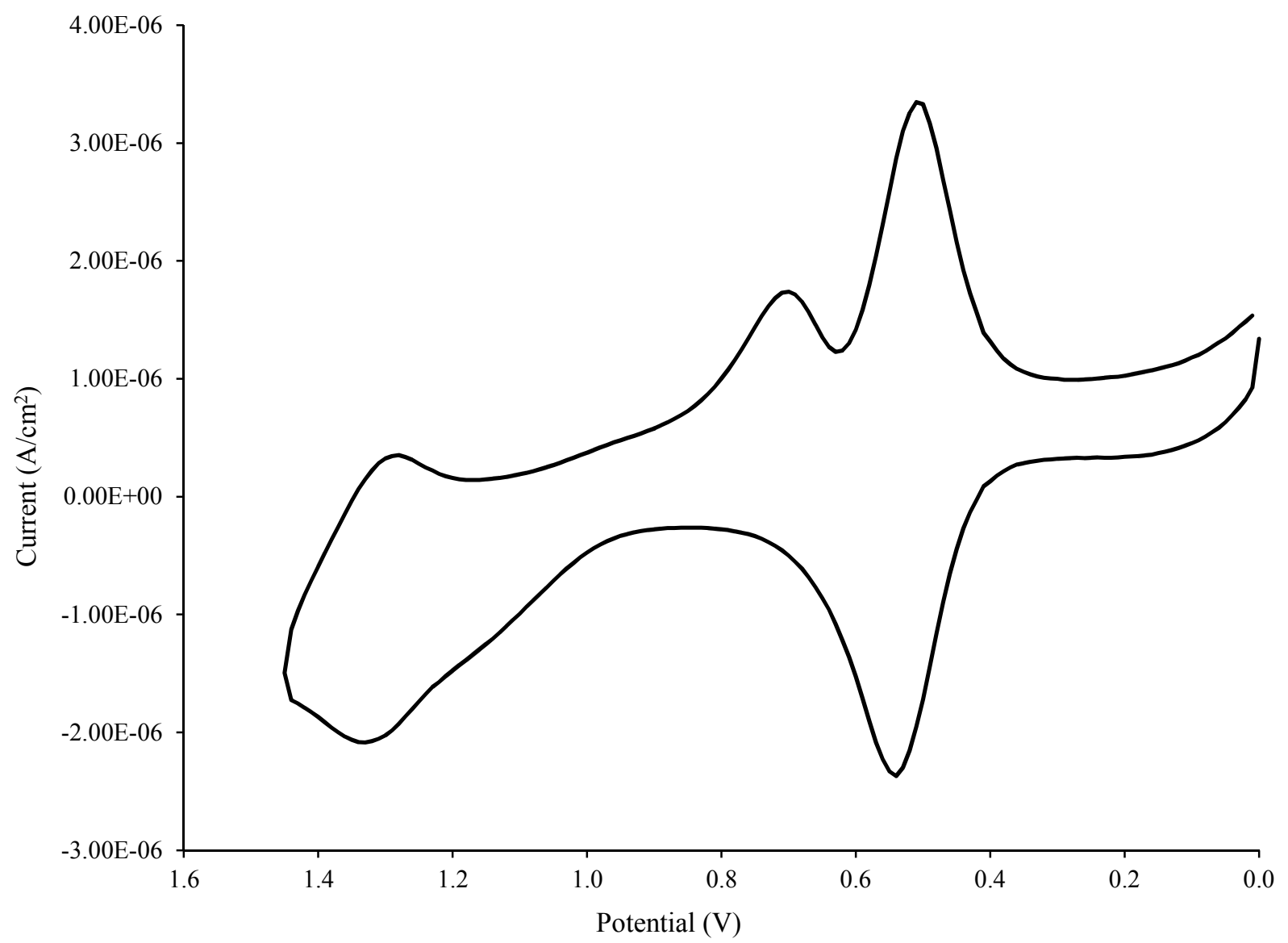

Figure S3. CV of $-\mathbf{R u P}_{\mathbf{3}}{ }^{2+}-\mathrm{Zr}(\mathrm{IV})-\mathbf{1}$ on planar FTO in a $\mathrm{pH} 1,0.1 \mathrm{M} \mathrm{HClO}_{4}, \mathrm{~V}$ vs. $\mathrm{Ag} / \mathrm{AgCl}, \mathrm{v}=0.025$ V/s. 

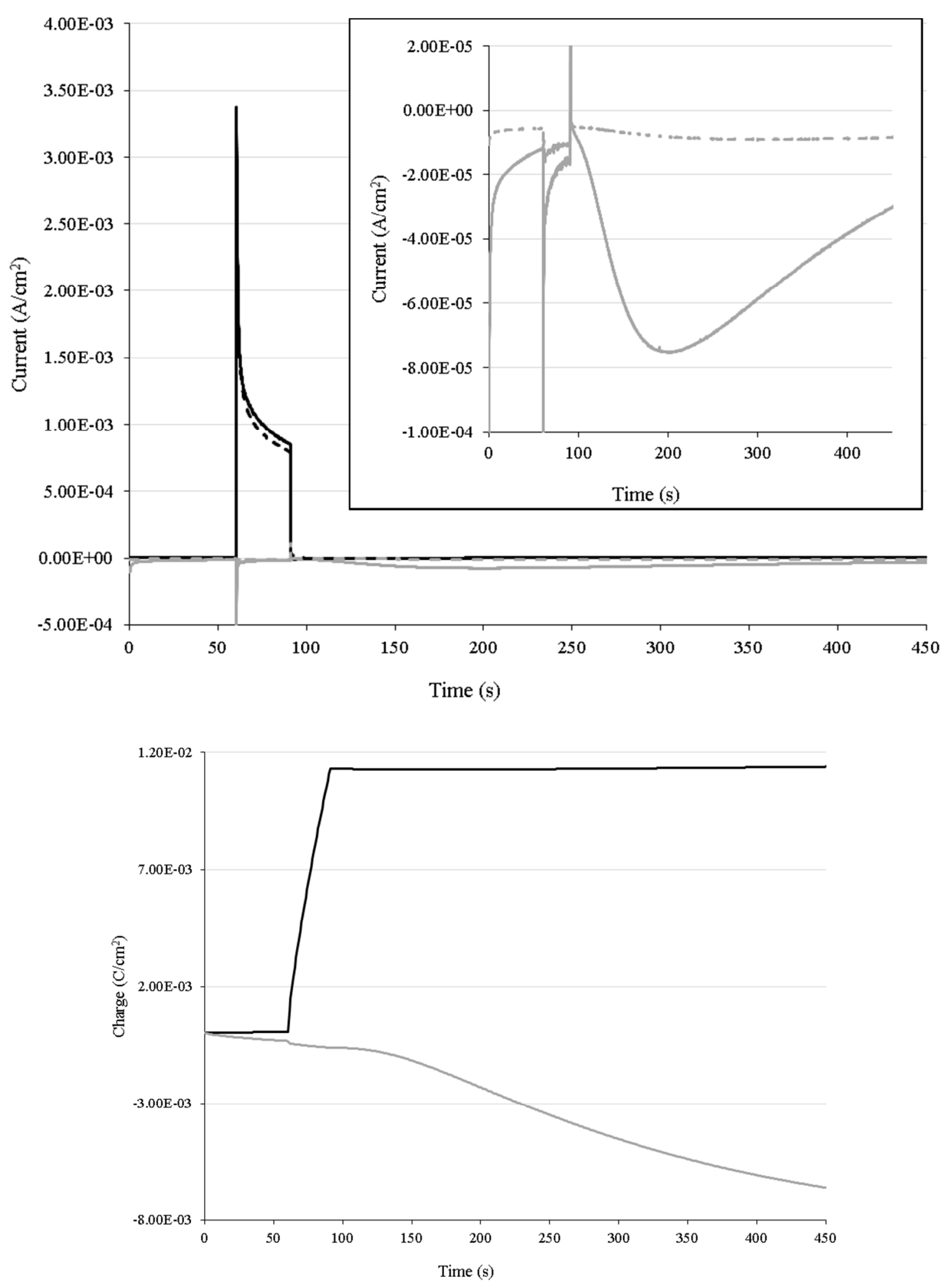

Figure S4. (top) I-t curves of a C-G cell of $\mathbf{R u P}-\mathbf{H}^{2+}$ co-loaded with $\mathbf{1}$ on $\mathrm{SnO}_{2} @ \mathrm{TiO}_{2}(4.3 \mathrm{~nm}) 5.5$ micron slides in pH 5.7, 0.1M acetate buffer, $1.0 \mathrm{M} \mathrm{NaClO}_{4}, \mathrm{E}_{\mathrm{app}, \mathrm{G}}=0.1 \mathrm{~V}, \mathrm{E}_{\mathrm{app}, \mathrm{C}}=-0.85 \mathrm{~V}$ (solid lines), $\mathrm{E}_{\mathrm{app}, \mathrm{C}}=$ $0.35 \mathrm{~V}$ (dashed lines), 1 Sun illumination from $\mathrm{t}=60-90 \mathrm{~s}$; (inset) current at the collector electrode.

Faradaic efficiency was $86 \%$. (bottom) Q-t curves for the experiment run with $\mathrm{E}_{\mathrm{app}, \mathrm{C}}=-0.85 \mathrm{~V}$ in the top figure, charge at the generator (black) and charge at the collector (gray). 


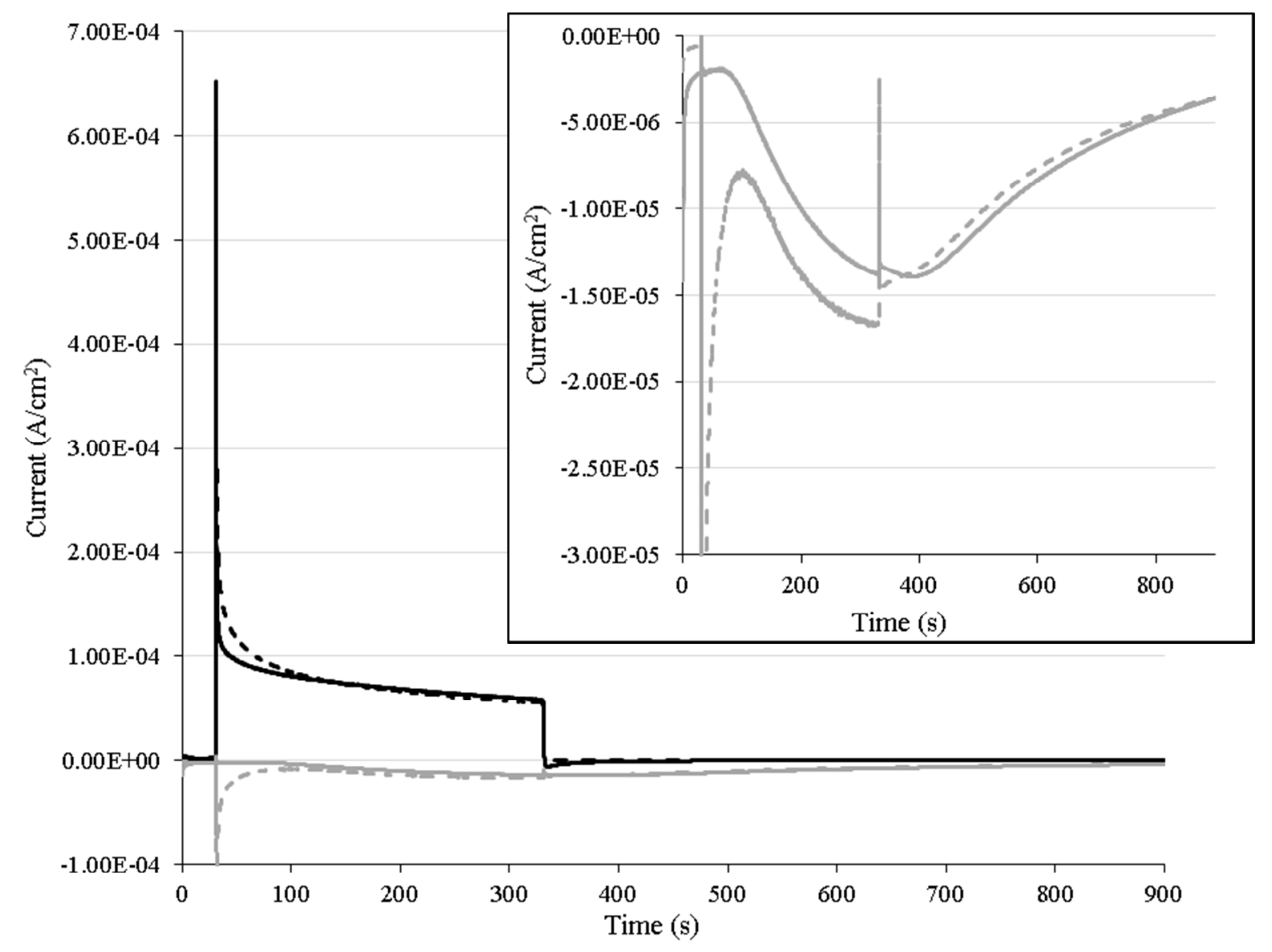

Figure S5. I-t curves of a C-G cell of RuP-H ${ }^{2+}$ co-loaded with 1 on $\mathrm{SnO}_{2} @ \mathrm{TiO}_{2}(4.3 \mathrm{~nm}) 5.5$ micron slide in $\mathrm{pH} 5.7,0.1 \mathrm{M}$ acetate buffer, with (solid lines) and without (dashed lines) $0.5 \mathrm{M} \mathrm{NaClO}_{4}, \mathrm{E}_{\mathrm{app}, \mathrm{G}}=0.1 \mathrm{~V}$, $\mathrm{E}_{\text {app }, \mathrm{C}}=-0.85 \mathrm{~V}, \sim 0.2$ Sun illumination from $\mathrm{t}=30-330 \mathrm{~s}$; (inset) current at the collector electrode.

Faradaic efficiency was $76 \%$ in the $0.5 \mathrm{M} \mathrm{NaClO}_{4}$ run. 


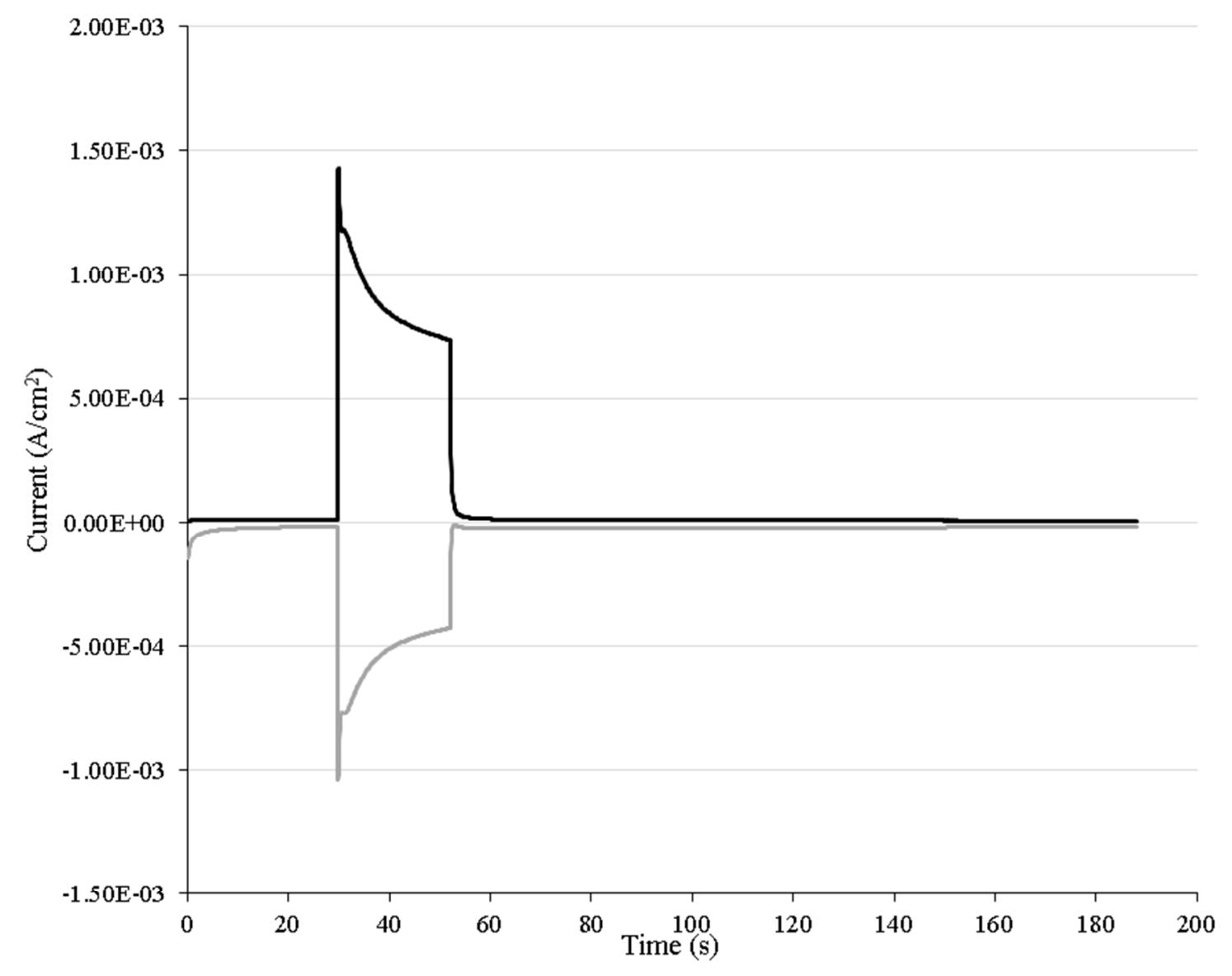

Figure S6. I-t curves of a C-G cell of $\mathbf{R u P - \mathbf { H } ^ { 2 + }}$ co-loaded with $\mathbf{1}$ on $\mathrm{SnO}_{2} @ \mathrm{TiO}_{2}(4.3 \mathrm{~nm}) 5.5$ micron slide in propylene carbonate with $8 \% \mathrm{pH} 5.7,0.1 \mathrm{M}$ acetate buffer, $0.2 \mathrm{M} \mathrm{NaClO}_{4}$ added, $\mathrm{E}_{\text {app, }, \mathrm{G}}=0.1 \mathrm{~V}, \mathrm{E}_{\mathrm{app}, \mathrm{C}}=-$ $0.85 \mathrm{~V}, 1$ Sun illumination from $\mathrm{t}=30-55 \mathrm{~s}$. 


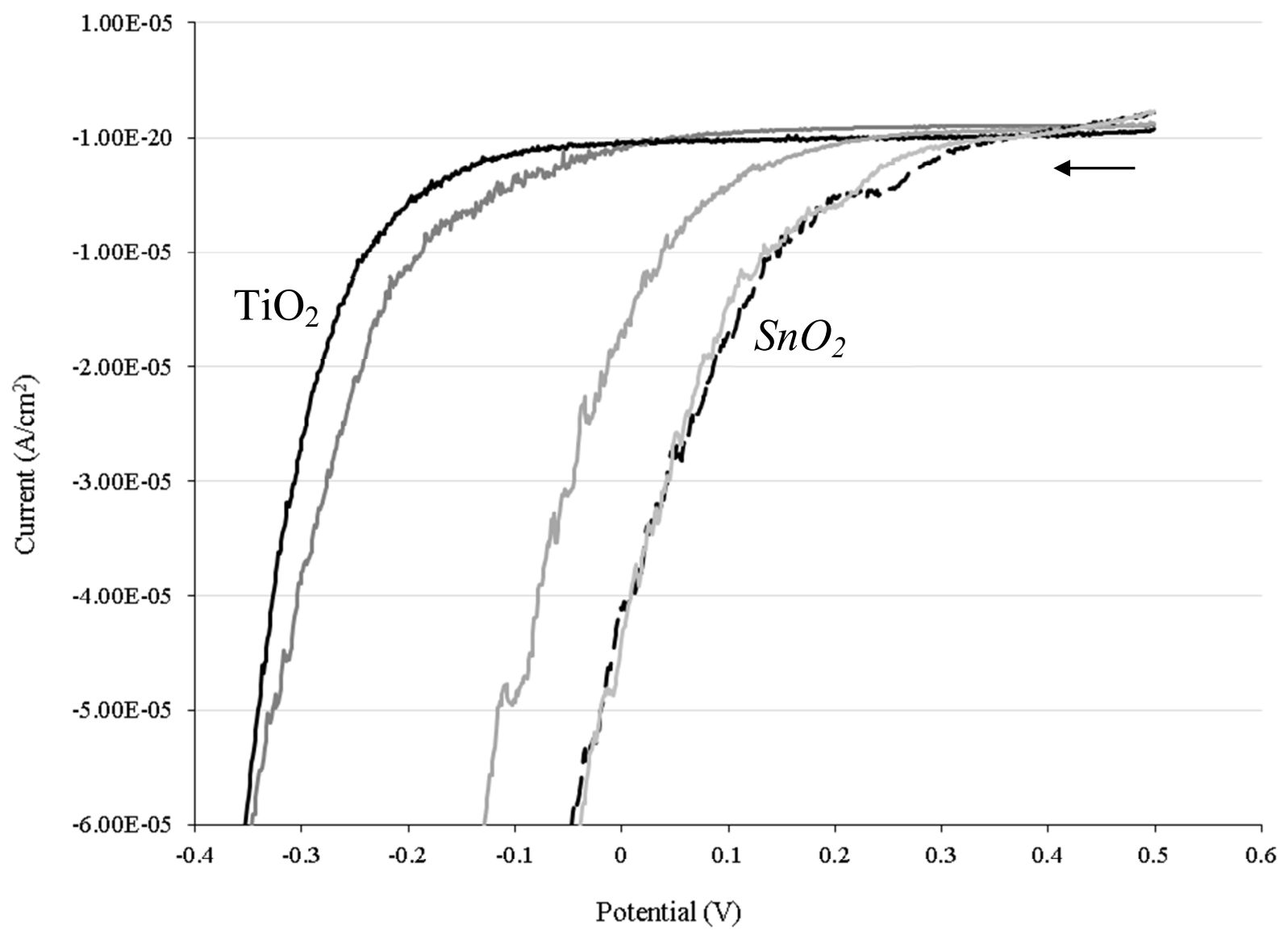

Figure S7. LSVs of $\mathrm{SnO}_{2}$ (--- black), $\mathrm{TiO}_{2}$ (black), and $\mathrm{SnO}_{2} @ \mathrm{TiO}_{2}$ core-shell electrodes with 25, 50, and 75 cycles of $\mathrm{TiO}_{2}$ (light to dark gray). 


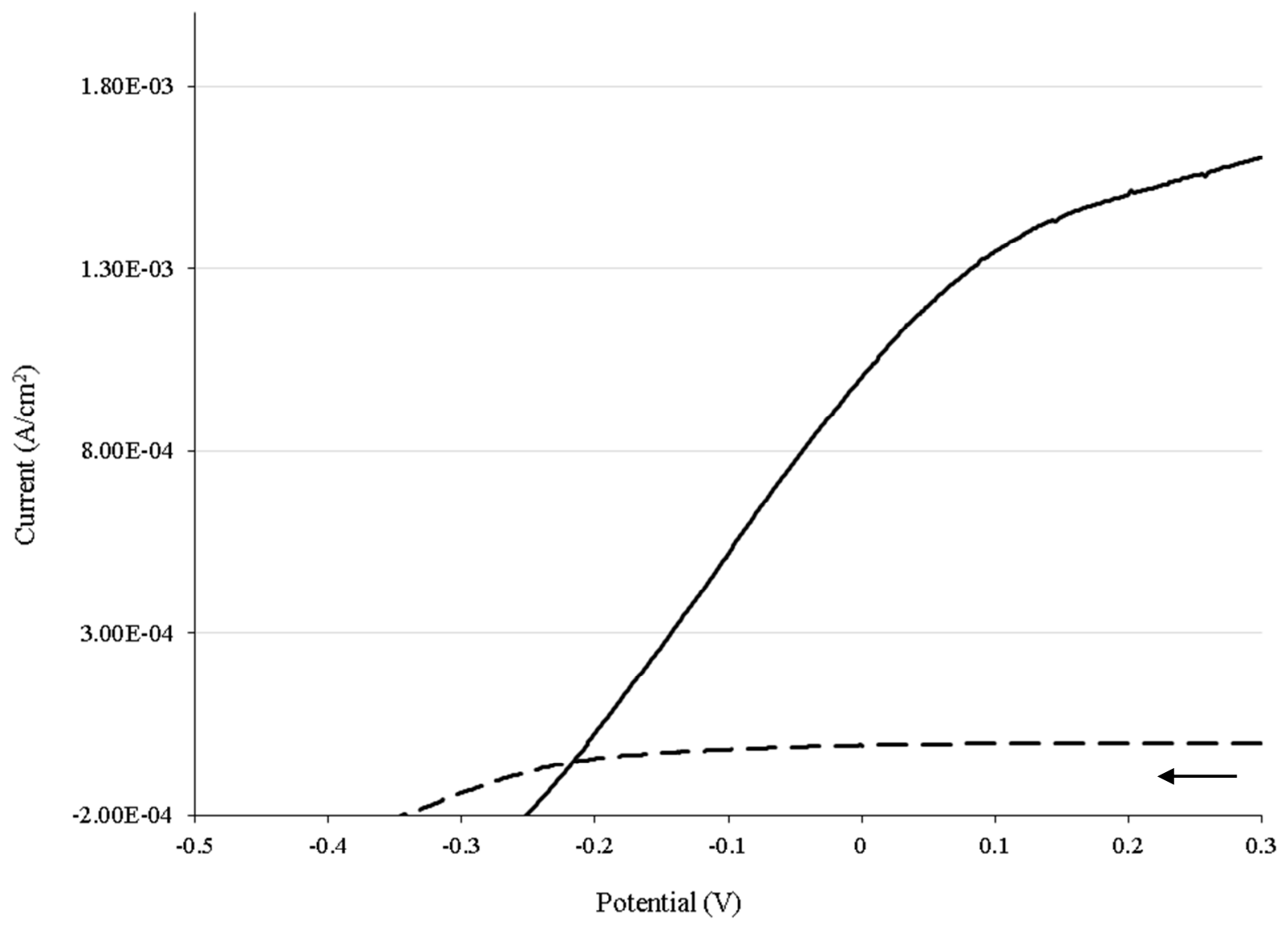

Figure S8. LSVs of $\mathbf{R u P}_{3}{ }^{2+}-\mathrm{Zr}(\mathrm{IV})-\mathbf{1}$ on $\mathrm{SnO}_{2} @ \mathrm{TiO}_{2}(4.3 \mathrm{~nm}) 5.5$ micron slide in the light (-black) and dark (--- black) in pH 5.7, 0.1M acetate buffer, $0.5 \mathrm{M} \mathrm{NaClO}_{4}, \mathrm{v}=0.05 \mathrm{~V} / \mathrm{s}$. 

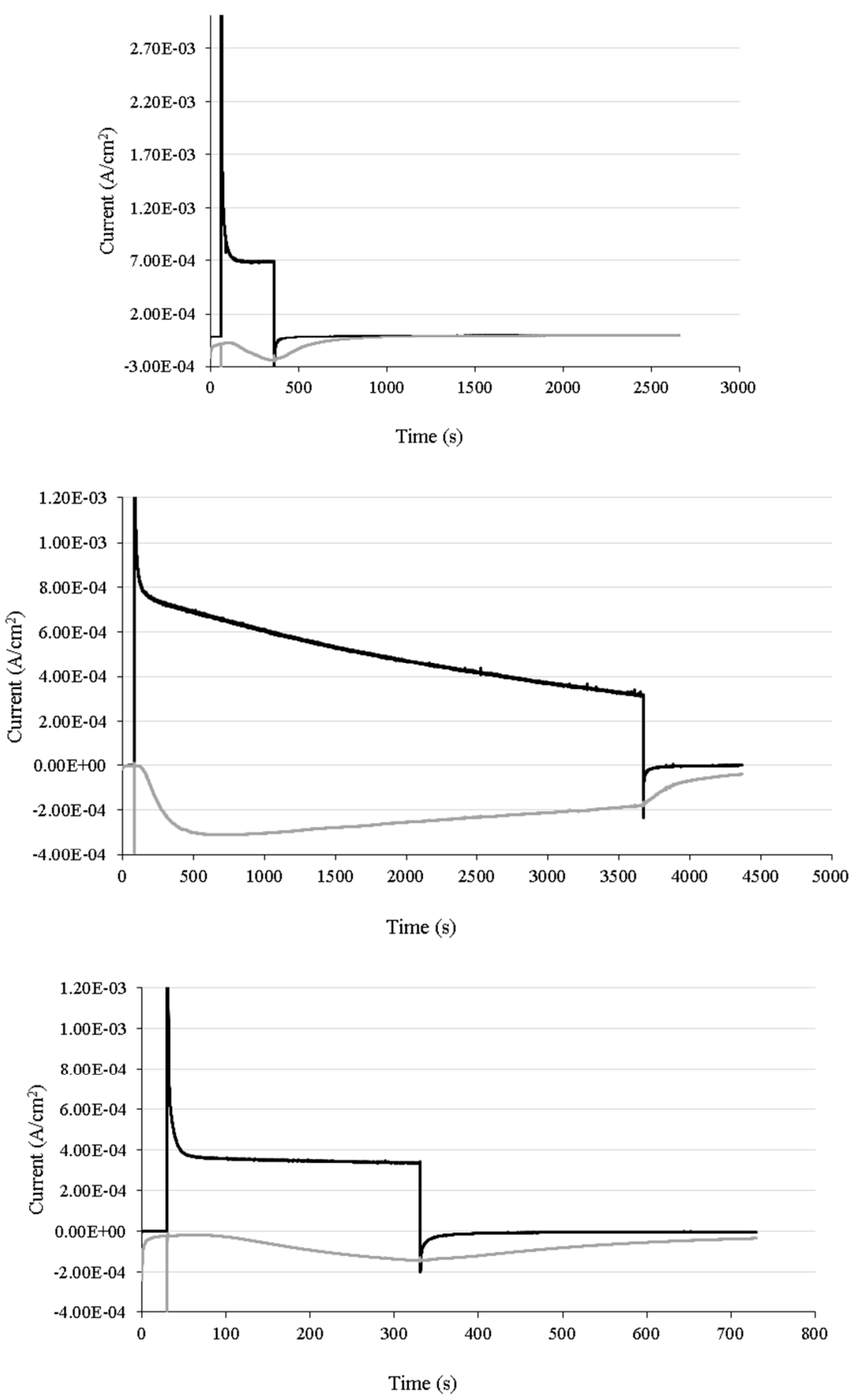

Figure S9 (top). I-t curves of a C-G cell of $\mathbf{R u C} \mathbf{H}_{2} \mathbf{P}_{\mathbf{2}}{ }^{2+}-\mathrm{Zr}-\mathbf{1}$ on $\mathrm{SnO}_{2} @ \mathrm{TiO}_{2}(4.3 \mathrm{~nm}) 5.5$ micron slide in pH 5.7, $0.1 \mathrm{M}$ acetate buffer, no $\mathrm{NaClO}_{4}$ present, $\mathrm{E}_{\text {app, } \mathrm{G}}=0.1 \mathrm{~V}, \mathrm{E}_{\text {app }, \mathrm{C}}=-0.85 \mathrm{~V}, 1$ Sun illumination from $\mathrm{t}$ $=60-3600$ s. (middle). I-t curves of a C-G cell of $\mathbf{R u C H} \mathbf{H}_{2} \mathbf{P}_{2}{ }^{2+}-\mathrm{Zr}-\mathbf{1}$ on $\mathrm{SnO}_{2} @ \mathrm{TiO}_{2}(4.3 \mathrm{~nm}) 5.5$ micron slide in $\mathrm{pH} 5.7,0.1 \mathrm{M}$ acetate buffer, no $\mathrm{NaClO}_{4}$ present, $\mathrm{E}_{\mathrm{app}, \mathrm{G}}=0.1 \mathrm{~V}, \mathrm{E}_{\mathrm{app}, \mathrm{C}}=-0.85 \mathrm{~V}, 1$ Sun illumination from $t=60-3600$ s. (bottom). I-t curves of a C-G cell of $\mathbf{R u C} \mathbf{H}_{2} \mathbf{P}_{\mathbf{2}}{ }^{2+}-\mathrm{Zr}-\mathbf{1}$ on $\mathrm{SnO}_{2} @ \mathrm{TiO}_{2}(4.3 \mathrm{~nm}) 5.5$ micron slide in $\mathrm{pH}$ 5.7, 0.1M acetate buffer, no $\mathrm{NaClO}_{4}$ present, $\mathrm{E}_{\mathrm{app}, \mathrm{G}}=0.1 \mathrm{~V}$, $\mathrm{E}_{\text {app,C }}=-0.85 \mathrm{~V}, 1$ Sun illumination from $\mathrm{t}=60-3600 \mathrm{~s}$. 


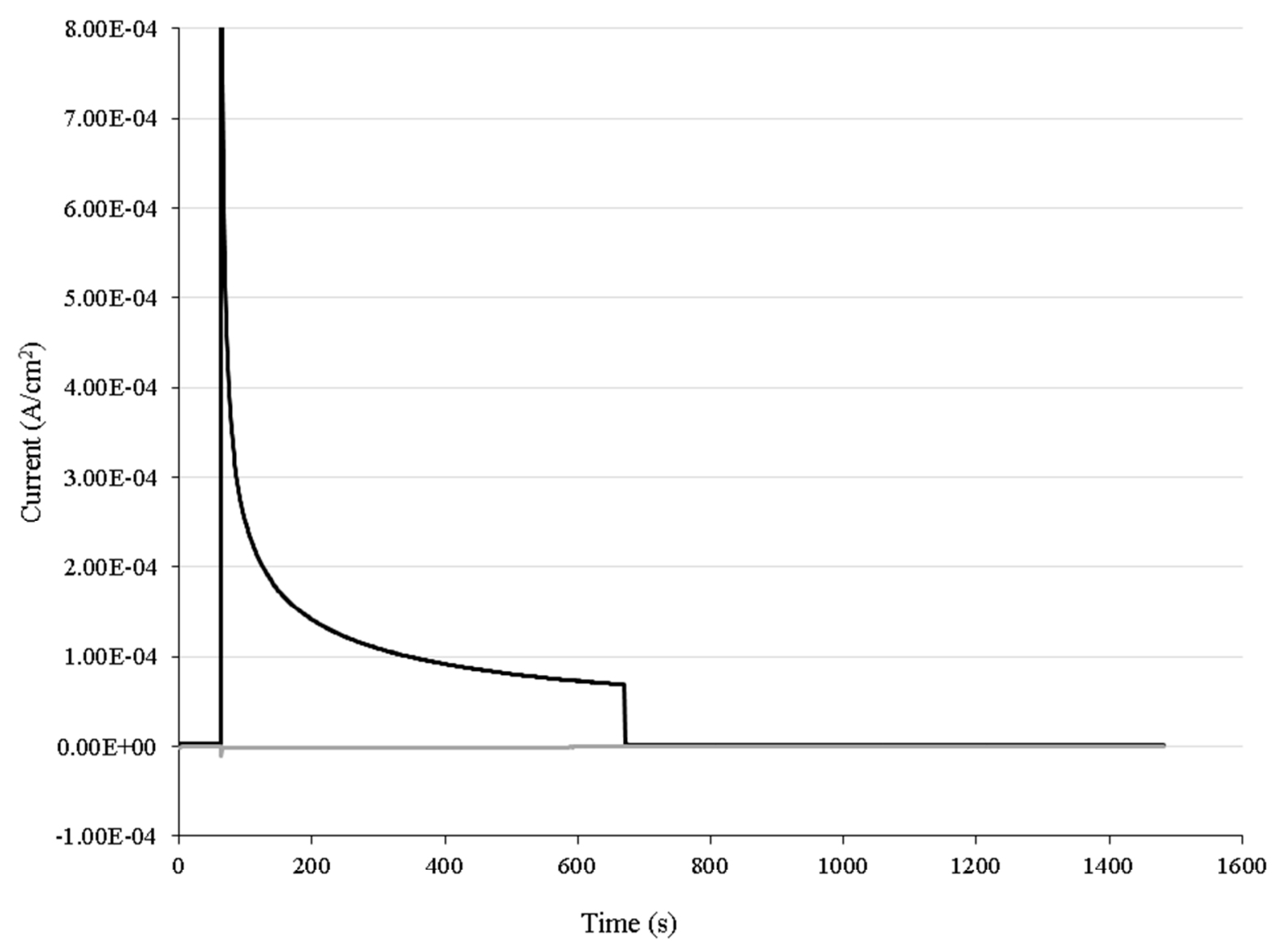

Figure S10. I-t curves of a C-G cell of RuP-H ${ }^{2+}$ on $\mathrm{SnO}_{2} @ \mathrm{TiO}_{2}(4.3 \mathrm{~nm}) 5.5$ micron slide in pH 5.7, $0.1 \mathrm{M}$ acetate buffer, with $0.5 \mathrm{M} \mathrm{NaClO}_{4}, \mathrm{E}_{\text {app }, \mathrm{G}}=0.1 \mathrm{~V}, \mathrm{E}_{\text {app, },}=-0.85 \mathrm{~V}, 1$ Sun illumination from $\mathrm{t}=60-$ $660 \mathrm{~s}$.

\section{$\underline{\text { References. }}$}

(1) Sherman, B. D.; Ashford, D. L.; Lapides, A. M.; Sheridan, M. V.; Wee, K.-R.; Meyer, T. J., LightDriven Water Splitting with a Molecular Electroassembly-Based Core/Shell Photoanode. J. Phys. Chem. Lett. 2015, 6, 3213-3217.

(2) Sheridan, M. V.; Sherman, B. D.; Marquard, S. L.; Fang, Z.; Ashford, D. L.; Wee, K.-R.; Gold, A. S.; Alibabaei, L.; Rudd, J. A.; Coggins, M. K., et al., Electron Transfer Mediator Effects in Water Oxidation Catalysis by Solution and Surface-Bound Ruthenium Bpy-Dicarboxylate Complexes. J. Phys. Chem. C 2015, 119, 25420-25428.

(3) Ashford, D. L.; Brennaman, M. K.; Brown, R. J.; Keinan, S.; Concepcion, J. J.; Papanikolas, J. M.; Templeton, J. L.; Meyer, T. J., Varying the Electronic Structure of Surface-Bound Ruthenium(II) Polypyridyl Complexes. Inorg. Chem. 2015, 54, 460-469.

(4) Zigler, D. F.; Morseth, Z. A.; Wang, L.; Ashford, D. L.; Brennaman, M. K.; Grumstrup, E. M.; Brigham, E. C.; Gish, M. K.; Dillon, R. J.; Alibabaei, L., et al., Disentangling the Physical Processes 
Responsible for the Kinetic Complexity in Interfacial Electron Transfer of Excited Ru(II) Polypyridyl Dyes on $\mathrm{TiO}_{2}$. J. Am. Chem. Soc. 2016, 138, 4426-4438.

(5) Norris, M. R.; Concepcion, J. J.; Glasson, C. R. K.; Fang, Z.; Lapides, A. M.; Ashford, D. L.;

Templeton, J. L.; Meyer, T. J., Synthesis of Phosphonic Acid Derivatized Bipyridine Ligands and Their Ruthenium Complexes. Inorg. Chem. 2013, 52, 12492-12501.

(6) Chappel, S.; Zaban, A., Nanoporous Sno2 Electrodes for Dye-Sensitized Solar Cells: Improved Cell Performance by the Synthesis of $18 \mathrm{~nm} \mathrm{SnO}_{2}$ Colloids. Sol. Energy Mater. Sol. Cells 2002, 71, 141-152. 